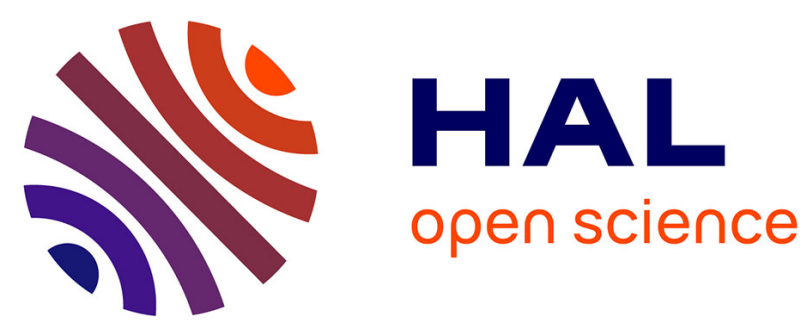

\title{
Investigation of Azimuthal Variations From X-Band Medium-Grazing-Angle Sea Clutter
}

Zaynab Guerraou, Sébastien Angelliaume, Luke Rosenberg, Charles-Antoine Guérin

\section{- To cite this version:}

Zaynab Guerraou, Sébastien Angelliaume, Luke Rosenberg, Charles-Antoine Guérin. Investigation of Azimuthal Variations From X-Band Medium-Grazing-Angle Sea Clutter. IEEE Transactions on Geoscience and Remote Sensing, 2016, 54 (10), p. 6110 - 6118. 10.1109/TGRS.2016.2581181 . hal01384263

\section{HAL Id: hal-01384263 https://hal.science/hal-01384263}

Submitted on 19 Oct 2016

HAL is a multi-disciplinary open access archive for the deposit and dissemination of scientific research documents, whether they are published or not. The documents may come from teaching and research institutions in France or abroad, or from public or private research centers.
L'archive ouverte pluridisciplinaire HAL, est destinée au dépôt et à la diffusion de documents scientifiques de niveau recherche, publiés ou non, émanant des établissements d'enseignement et de recherche français ou étrangers, des laboratoires publics ou privés. 


\title{
Investigation of azimuthal variations from X-band medium grazing angle sea clutter
}

\author{
Zaynab Guerraou, Sébastien Angelliaume, Luke Rosenberg and Charles-Antoine Guérin
}

\begin{abstract}
The Ingara X-band fully-polarimetric medium grazing angle sea clutter data set was collected in the Australian maritime environment over an angular range of $360^{\circ}$ in azimuth and $15^{\circ}$ to $45^{\circ}$ in grazing. This paper reports further analysis of this data set, focussing on understanding the azimuth variation to enable improved simulation accuracy and extraction of relevant geophysical parameters. This includes some original properties of the co- and cross-polarized normalized radar cross section as a function of the scattering geometry and sea surface parameters. We also assess the performances and limitations of recent sea surface scattering models in the light of this rich data set.
\end{abstract}

Index Terms-Normalized radar cross section (NRCS), polarization, azimuthal variation, sea surface, medium grazing angles

\section{INTRODUCTION}

The study and modeling of the sea surface clutter in the microwave regime is important for both maritime surveillance (i.e. detecting targets in sea clutter) and for better understanding the maritime environment (i.e. the recovery of geophysical parameters). This latter application has reached a mature state in the last decades with the simultaneous improvements of analytic scattering models [1], descriptions of the sea surface [2]-[4] and the ever increasing capabilities of airborne and space-borne instruments [5]. In most cases, the sea surface and scattering models provide a satisfactory agreement with the experimental observations, both from the qualitative and quantitative point of view (see [6]-[10] for the assessment of some recent airborne radar data and [11]-[13] for spaceborne data). However, some issues remain in understanding and modeling the effects of microwave ocean scattering. These include difficulties in characterising the cross-polarized and horizontally-polarized return, the variability of the normalized radar cross section (NRCS) due to 'forcing' parameters (mixed sea systems, swell, currents, wave age, etc.), the influence of breaking waves, the directional wave number spectrum of short-waves, and azimuthal variations including directional asymmetry. In this paper, we will investigate this final issue using the Ingara $\mathrm{X}$-band medium grazing angles (MGA) sea clutter data set which was collected by the Defence Science and Technology Group [14]. This data set is described in Section II with an original technique presented in Section III for processing data with weak signal (i.e. clutter) to noise ratios (SNR).

Z. Guerraou and S. Angelliaume are with the ONERA, Département Electromagnétisme et Radar, France.

L. Rosenberg is with the Defence Science and Technology Group, Australia.

C.-A. Guérin is with the Université de Toulon at the Mediterranean Institute of Oceanography (MIO, UM 110 UTLN/AMU/CNRS-INSU/IRD) F-83957 La Garde, France.
Numerous data sets described in the literature show evidence of azimuthal variation of the NRCS at MGAs with different empirical relationships derived to relate the angular variations to the wind and/or wave directions [15]-[17].

Rather than propose a new geophysical 'model function' for sea clutter returns, this work is concerned with the characterization of the azimuthal variations and directional asymmetries observed in sea-clutter. The results inferred from this study should eventually be confirmed on other datasets, with the long term objective being to incorporate them into a physical model. Such an improved model would be useful in estimating the main geophysical parameters such as wind speed and direction, significant wave height and wave age.

In Section IV, we investigate the azimuthal variations of the NRCS for both the horizontal $(\mathrm{H})$ and vertical $(\mathrm{V})$ channels. A vanishing of the secondary downwind maximum is observed with the $\mathrm{HH}$ polarization at the lowest grazing angle $\left(15^{\circ}\right)$, thereby confirming the phenomenon observed and modelled in the empirical Georgia Institute of Technology (GIT) mean backscatter model [18]. The directional asymmetries are shown to be polarization-dependent and follow non-monotonic variations with respect to the grazing angle.

In Section V, the relationships between the different polarization channels are investigated in terms of the underlying physical scattering mechanisms. Section VI then shows a comparison of the angular variations of the fully-polarized data set with recent scattering and directional sea spectrum models. We show that the agreement with data can be improved by resorting to these recent sea surface scattering models, although there remains a number of observed features which are not correctly modeled.

\section{The INGARA MGA SEA CLUTTER DATA SET}

Ingara is a fully-polarimetric X-band radar system maintained within the DST Group in Australia [19]. During the MGA sea-clutter trials, the central frequency was set to 10.1 $\mathrm{GHz}$ and the radar collected real aperture $\mathrm{I} / \mathrm{Q}$ data with range and azimuth resolutions of $0.75 \mathrm{~m}$ and $63 \mathrm{~m}$ respectively. As shown in Fig. 1, data were collected over a range of sea conditions using a circular spotlight collection with grazing angles spanning $15^{\circ}$ to $45^{\circ}$. The total collection across all grazing angles for a single run took approximately 90 minutes and it is not unreasonable to assume that the ocean surface conditions remain relatively unchanged. The MGA sea clutter trials were conducted over several days in two distinct maritime regions. The first 'sea cutter trial' (SCT04) occurred in 2004 over the Southern Ocean, approximately $100 \mathrm{~km}$ south 
of Port Lincoln, South Australia. Eight days of data were collected with environmental conditions summarised in Table I (runs 1-8). A further four days of data were collected during the second 'maritime sea clutter trial' (MAST06) (runs 9-12). This trial was conducted in 2006 over littoral and open ocean environments near Darwin in the Northern Territory. The sea conditions were measured using DST Group's Tri-axys wave buoy which was located within $50 \mathrm{~km}$ of the imaging site. Wind data from several different sources were collected and compared during the trials. For the SCT04, the most reliable wind data were obtained from the Bureau of Meteorology (BoM) automatic weather station located on a cliff top about $50 \mathrm{~km}$ north-east of the wave buoy deployment site. Hence it will be subject to some error and possibly some time delay compared to the actual conditions at the wave buoy site. For MAST06, the most reliable wind data were made with a handheld anemometer on a boat near the imaging area except for one collection where the wind vector predicted by the Australian BoM Meso-scale Limited Area Prediction System (LAPS) model was used [14]. The Douglas sea state reported in Table I is based on the wave height. Figure 2 shows an example of the radar backscatter for $\mathrm{HH}$ polarization of run day 3 with a wind speed of $10.3 \mathrm{~m} / \mathrm{s}$ and a wave height of $2.6 \mathrm{~m}$. The data set was collected over a full 360 degree orbit of the scene and has been rotated so that upwind is at $0^{\circ}$. It is noteworthy that the imaged scenes contain waves driven from both wind and underlying swells. This is illustrated by the patterns observed in Fig. 2 which correspond to swell modulations. Although it was difficult to isolate the different wave and wind effects, the analysis performed helps to draw out interesting trends in the data.

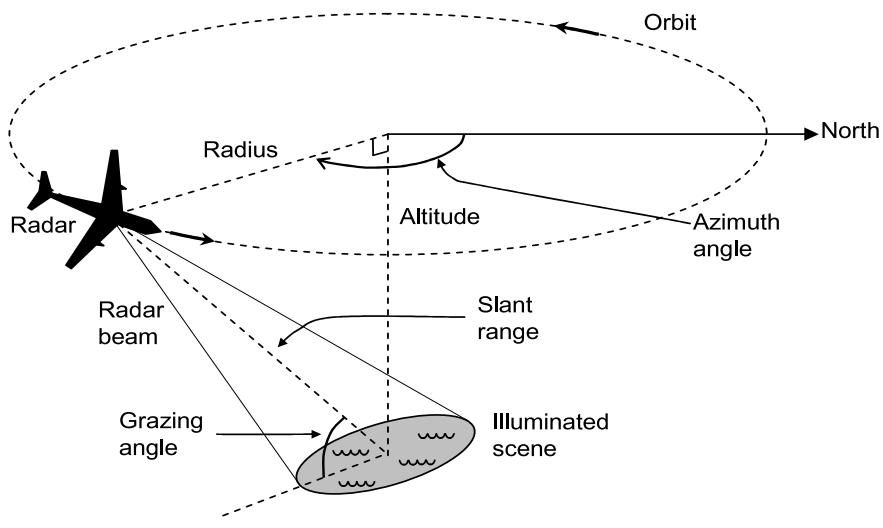

Fig. 1: Circular spotlight mode collection geometry.

In Fig. 3, significant wave heights, $H_{s}$, from the Ingara MGA data set are compared to those inferred from the classical Elfouhaily (Elf) spectral model [3] used in unlimited fetch conditions. As can be seen, the $H_{s}$ values reported in the SCT04 campaign are much larger than those predicted by a fully-developed wind-sea surface, $H_{s} \simeq 0.025 U^{2}$ where $U$ is the wind speed at $10 \mathrm{~m}$ height, indicating the likely presence of large amplitude swell. On the contrary, the low $H_{s}$ values observed for the MAST06 experiment are explained by data being subject to a daily wind cycle where the wind duration was constant for only a few hours in any given direction. This implies the seas during MAST06 were fully-developed only

\begin{tabular}{|l|l|l|l|l|l|l|}
\hline \multirow{2}{*}{$\begin{array}{l}\text { Run } \\
\text { day }\end{array}$} & \multirow{2}{*}{$\begin{array}{l}\text { Douglas } \\
\text { sea state }\end{array}$} & \multicolumn{2}{|c|}{ Wind } & \multicolumn{3}{c|}{ Wave } \\
\cline { 3 - 7 } & & $\begin{array}{l}\text { Speed } \\
(\mathrm{m} / \mathrm{s})\end{array}$ & $\begin{array}{l}\text { Direction } \\
(\mathrm{deg})\end{array}$ & $\begin{array}{l}\text { Height } \\
(\mathrm{m})\end{array}$ & $\begin{array}{l}\text { Direction } \\
(\mathrm{deg})\end{array}$ & $\begin{array}{l}\text { Period } \\
(\mathrm{s})\end{array}$ \\
\hline 1 & 6 & 10.2 & 248 & 4.9 & 220 & 12.3 \\
\hline 2 & 5 & 7.9 & 248 & 3.5 & 205 & 11.8 \\
\hline 3 & 5 & 10.3 & 315 & 2.6 & 210 & 10.4 \\
\hline 4 & 5 & 13.6 & 0 & 3.2 & 293 & 8.8 \\
\hline 5 & $4-5$ & 9.3 & 68 & 2.5 & 169 & 9.7 \\
\hline 6 & 5 & 9.5 & 315 & 3.0 & 234 & 11.4 \\
\hline 7 & 6 & 13.2 & 22 & 3.8 & 254 & 12.2 \\
\hline 8 & 7 & 8.5 & 0 & 4.3 & 243 & 12.5 \\
\hline 9 & 3 & 8.5 & 115 & 0.62 & 112 & 3.1 \\
\hline 10 & 2 & 3.6 & 66 & 0.25 & 35 & 2.6 \\
\hline 11 & 2 & 3.5 & 83 & 0.41 & 46 & 4.0 \\
\hline 12 & 3 & 10.2 & 124 & 1.21 & 128 & 4.6 \\
\hline
\end{tabular}

TABLE I: Wind and wave ground truth for the Ingara MGA data. Run days 18 are from the SCT04 trial, while 9-12 are from MAST06. Douglas sea state is determined by the wave height.

at very low wind speeds.

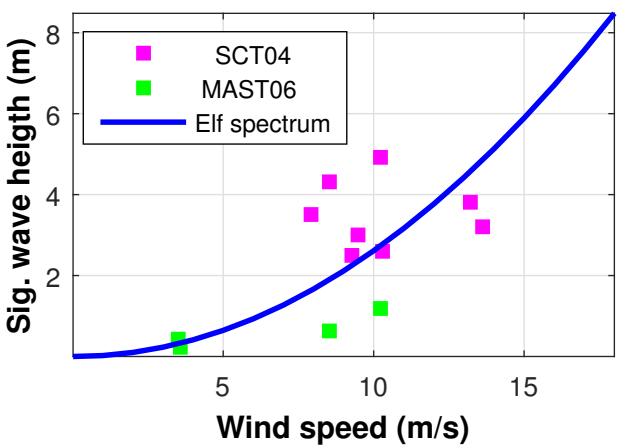

Fig. 3: Significant wave height with respect to wind speed as derived from Elfouhaily spectrum and the measured environmental conditions during the Ingara MGA trials.

\section{DATA PROCESSING}

To estimate the sea-clutter NRCS, a number of preprocessing steps were applied to the collected data. Firstly, the sampled signal was adjusted for motion compensation using both the inertial navigation unit and the global positioning system onboard the radar platform. The next steps included a correction for the variation in ground range resolution due to changes in grazing angle, removal of the elevation beampattern and polarimetric calibration using trihedral corner reflectors on the ground, assuming that calibration parameters are constant during the flight. Motion compensation affected the phase of the radar backscatter, while adjustment for the ground range resolution altered the NRCS. The calibration was validated by verifying the stability of the radar cross talk, channel imbalance and absolute gains for several hours before the flight.

For the lower grazing angles and both $\mathrm{HH}$ and HV polarization channels, the SNR is low and an accurate denoising procedure is of primary importance for correct retrieval of the NRCS. In previous work [20], the mean instantaneous thermal noise power of the radar system was estimated by processing part of the collection where the transmitter was 


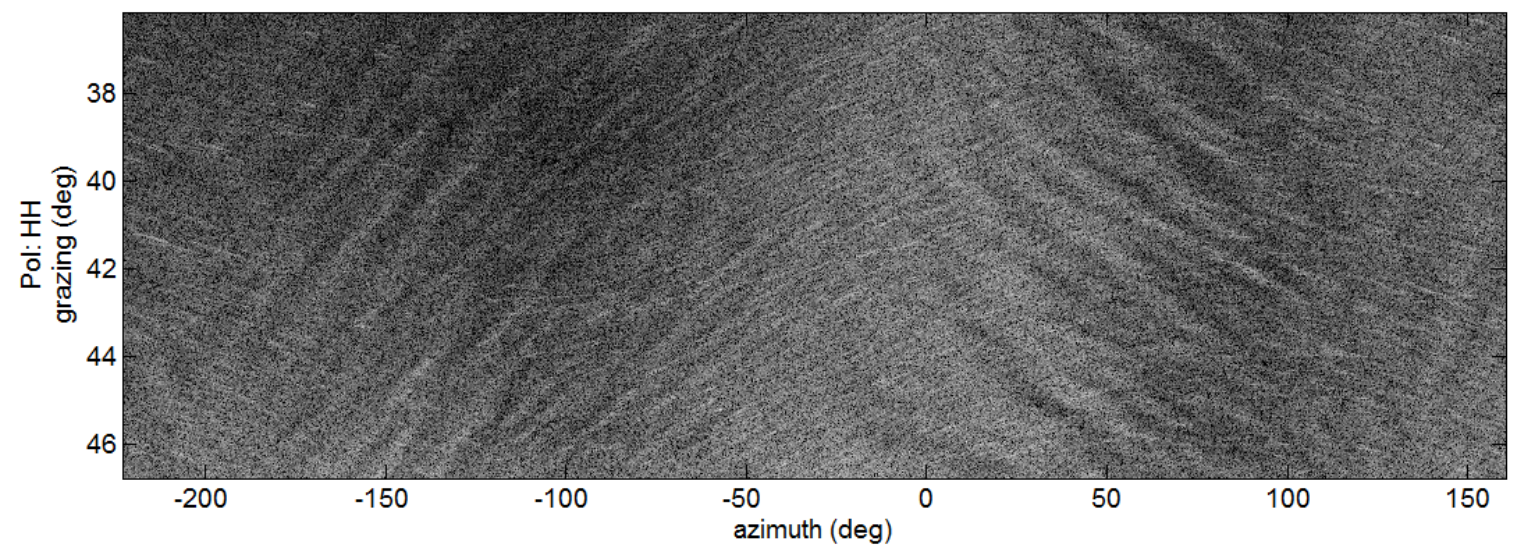

Fig. 2: Example of Ingara HH sea-clutter data as a function of azimuth angle.

turned off. By assuming the sea-clutter and thermal noise are independent throughout the processing chain, a noise signal was then created in the backscatter coefficient domain with the same pre-processing steps applied as the clutter plus noise signal. It is important to note that the noise level is constant within the radar. However, the noise component of the radar return after pre-processing and calibration becomes slightly larger at lower grazing angles. A point-by-point denoising by subtracting this noise from the measured data is not always appropriate as it may result in non-physical negative intensities and if these are rejected in the estimate of the NRCS, there is the possibility of introducing an artificial bias. To overcome these limitations and obtain an estimate of the mean noise power in the low SNR regions, we have therefore proposed a new probabilistic denoising approach based on a maximum likelihood estimator (MLE).

We assume an additive white noise on the recorded NRCS, with mean and variance depending on the grazing angle but not the azimuthal direction. This last assumption is well supported by experimental evidence.

For the double purpose of estimating the ensemble-averaged NRCS and reducing the noise, we average data in bins of $5^{\circ}$ in azimuth and $1^{\circ}$ in grazing with each bin containing approximatively $10^{6}$ samples. Then assuming that the statistical parameters describing both the noise and the NRCS do not vary appreciably in such a small angular domain, the ensemble-averaged NRCS at a given grazing angle can be written as a Fourier series describing the azimuth variation with an additive Gaussian background noise:

$$
\sigma_{0}^{\text {model }}\left(\phi_{n}\right)=\tilde{\sigma}_{0}\left(\phi_{n}\right)+b\left(\phi_{n}\right),
$$

where $\phi_{n}$ is the azimuth angle relative to the wind direction, $b\left(\phi_{n}\right)$ is a realization of the mean noise and $\tilde{\sigma}_{0}\left(\phi_{n}\right)$ is the denoised NRCS described by the following model:

$$
\tilde{\sigma}_{0}\left(\phi_{n}\right)=a_{0}+\sum_{k=1}^{4} a_{k} \cos \left(k\left(\phi_{n}-\delta_{k}\right)\right) .
$$

Four harmonics have been found sufficient to reproduce the mean azimuthal variations in the NRCS (See [21]). Also, the NRCS extrema do not always coincide with the cardinal directions (up/down/cross wind) and are sometimes significantly shifted from their expected position. Therefore, we did not assume any a-priori symmetry with respect to wind direction and have introduced additional phase parameters, $\delta_{n}$, in the cosine expansion. Note that the amplitude is an implicit function of polarization, grazing angle and sea state.

After averaging in each bin, the mean noise power can reasonably be described with a Gaussian distribution by virtue of the central limit theorem, even through it is known to follow an exponential distribution. Our analysis assumes that antenna and system noise are the dominant contributors to the noise estimates and that environment sources of noise variability with look direction (both azimuth and grazing angle) can be neglected. The antenna will contribute a grazing angle dependency in the system noise which has been estimated for the denoising process, while analysis has shown that the variation over azimuth is approximately constant and may be approximated with a constant mean, $\bar{b}$, and variance, $\sigma_{b}^{2}$. Also, since the denoised NRCS is deterministic, the overall NRCS model can be represented as a shifted normal distribution:

$$
\sigma_{0}^{\text {model }}\left(\phi_{n}\right) \sim \mathcal{N}\left(\tilde{\sigma}_{0}\left(\phi_{n}\right)+\bar{b}, \sigma_{b}^{2}\right) .
$$

Under these assumptions, the log-likelihood of the observations can be written as follows:

$$
\mathcal{L}=-\frac{1}{2} \sum_{n=1}^{N_{a}} \log \left(2 \pi \sigma_{b}^{2}\right)-\sum_{n=1}^{N_{a}} \frac{1}{2 \sigma_{b}^{2}}\left[\sigma_{0}^{\text {data }}\left(\phi_{n}\right)-\left(\tilde{\sigma}_{0}\left(\phi_{n}\right)+\bar{b}\right)\right]^{2},
$$

where $N_{a}$ is the number of bins in azimuth. This can also be rewritten after substituting the NRCS model in (III.2):

$$
\begin{aligned}
\mathcal{L}=- & \frac{1}{2} \sum_{n=1}^{N_{a}} \log \left(2 \pi \sigma_{b}^{2}\right)-\left(\sum _ { n = 1 } ^ { N _ { a } } \frac { 1 } { 2 \sigma _ { b } ^ { 2 } } \left[\sigma_{0}^{\text {data }}\left(\phi_{n}\right)\right.\right. \\
& \left.\left.-\left(a_{0}+\sum_{k=1}^{4} a_{k} \cos \left(k\left(\phi_{n}-\delta_{k}\right)\right)+\bar{b}\right)\right]^{2}\right) .
\end{aligned}
$$

The parameters $a_{k}$ and $\delta_{k}$ in the model NRCS can be obtained by maximization of the log-likelihood. This is performed by finding the set of values which cancel the partial derivatives $\frac{\partial \mathcal{L}}{\partial a_{k}}$ and $\frac{\partial \mathcal{L}}{\partial \delta_{k}}$. This leads to a linear system of size $9 \times 9$ for the 9 unknowns $\left(a_{0}, a_{k}, \delta_{k}\right)$, with matrix elements 
merely depending on the bin-averaged data $\sigma_{0}^{\text {data }}\left(\phi_{k}\right)$ and the mean noise power $\bar{b}$. Due to page constraints, the derivation is not reproduced here but is straightforward to obtain.

Figure 4 shows an example of the denoised NRCS, its comparison with the original data and the mean value of the azimuthal noise. As can be seen for low grazing angles where the SNR is low, the full dynamical range of the HH NRCS can only be seen after appropriate denoising.

To test the robustness of the estimation scheme, a validation procedure was performed by simulating the NRCS using the GO-SSA scattering model [22] introduced in Section VI and then adding different levels of simulated noise. The root mean square error calculated between the noise-free simulated data and the estimated model is found to be significantly low and quite insensitive to the SNR (It remains of the order of $10^{-9}$ for an SNR as low as $-30 \mathrm{~dB}$ ). Another possible source of mismatch between model and data is the limited number of azimuthal harmonics in the model. This was verified by simulating a 10-harmonic NRCS and then reconstructing it with only 4 harmonics. The results were still found to be accurate and robust to the SNR.
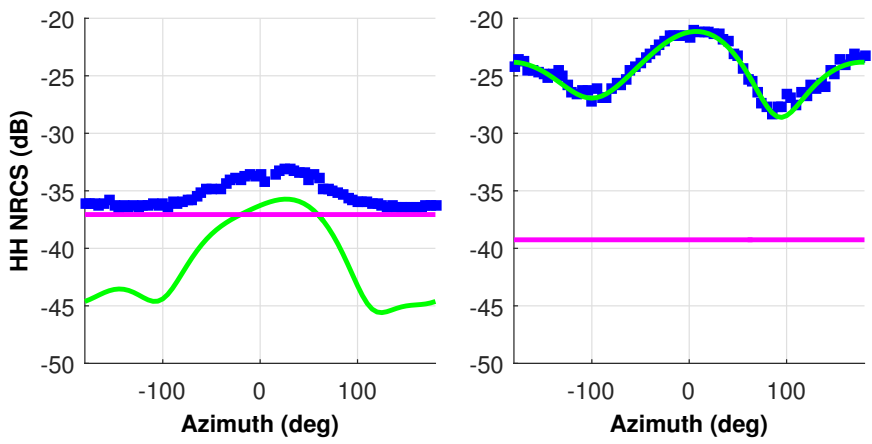

Fig. 4: Raw (ם) and denoised NRCS (green) for the HH polarization, run day 9 at grazing angles: $15^{\circ}$ (left) and $45^{\circ}$ (right). The mean noise estimate is shown in magenta. Note that the azimuth axis has been rotated so that $0^{\circ}$ is upwind.

\section{ANGULAR VARIATION OF THE NRCS}

\section{A. Grazing variations}

The MLE based fitting and denoising procedure has been systematically applied to the different run days for all grazing angles. Figure 5 shows an example of the co-polarized NRCS (HH and VV) for run day 9 as a function of the grazing angle for different azimuthal directions: upwind, crosswind and downwind. The noise estimate exhibits a variation of up to 4 $\mathrm{dB}$ with grazing angle but only the mean value for this specific run day is superimposed on the plots, with the remaining solid lines corresponding to a third-order polynomial model that has been fitted to the NRCS.

In this figure, the upwind and downwind returns in $\mathrm{VV}$ are close, while the crosswind return is lower by about $7 \mathrm{~dB}$. This is not the case in $\mathrm{HH}$ where the downwind and crosswind values are very close at the lowest grazing angles. In addition, the denoised NRCS in this region is up to $10 \mathrm{~dB}$ lower than the noise floor. We recall that the total collection across all grazing angles took approximately 90 minutes. Even though it seems reasonable to assume that the ocean surface conditions remain relatively stable over such short time intervals and that mean backscatter variations are for the most part related to the changing imaging geometry rather than changing ocean conditions, it is possible that this has caused some variations across grazing angles.
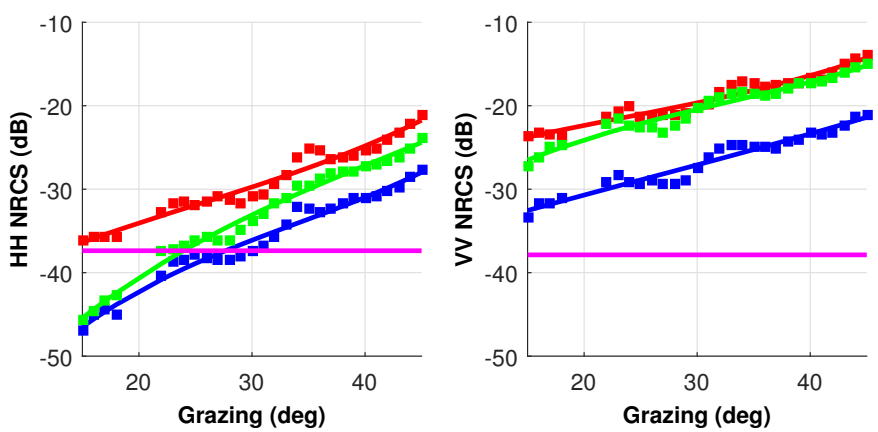

Fig. 5: Variation of the NRCS with grazing angle for run day 9 for $\mathrm{HH}$ (left) and VV (right) polarizations. Azimuth directions: upwind (red), crosswind (blue) and downwind (green). Mean noise estimate is shown in magenta.

\section{B. Azimuthal variations}

We now investigate the azimuthal variation after the MLE based denoising. Figure 6 illustrates the azimuthal variations of the resulting co-polarized NRCS (VV and $\mathrm{HH}$ ) for run day 9 (wind speed of $8.5 \mathrm{~m} / \mathrm{s}$ ) with various grazing angles. At moderate grazing angles we recover the commonly observed pattern of a sinusoidal variation with respect to the wind direction: a maximum in the upwind direction, a secondary maximum in the downwind direction and two minima in the crosswind directions. Note that the variations are sometimes asymmetric around the upwind direction which may be due to the occurrence of an extra swell system not aligned with the wind direction. This also can be linked to any potential wind measurement inaccuracy.

As the grazing angle is decreased in $\mathrm{HH}$ polarization, the angular distribution shifts progressively from two local maxima in the upwind/downwind direction to a unique and pronounced maximum in the upwind direction. The interpretation and physical modeling of this peculiar azimuthal behavior at low grazing angles are not easily established. To better characterize this observed behaviour, we have investigated the upwind/downwind asymmetry (UDA) and upwind/crosswind asymmetry (UCA). This is defined as the difference (in $\mathrm{dB}$ ) of the NRCS level at the cardinal directions for the same grazing angle.

Observed values of the maximum UDA and UCA in the $\mathrm{HH}$ and VV polarizations, together with the grazing angle where these maxima occur have been reported. Note that values for the two crosswind directions ( \pm 90 degrees) have been averaged as they sometimes exhibit different levels of NRCS. This is seen in Fig. 6 and is likely due to the presence of a different wave system (swell) in the sea.

From these results, the UDA and UCA for both polarizations reach their maximum at a moderate grazing angle between $35^{\circ}$ and $45^{\circ}$. This angle varies slightly with wind speed but no 

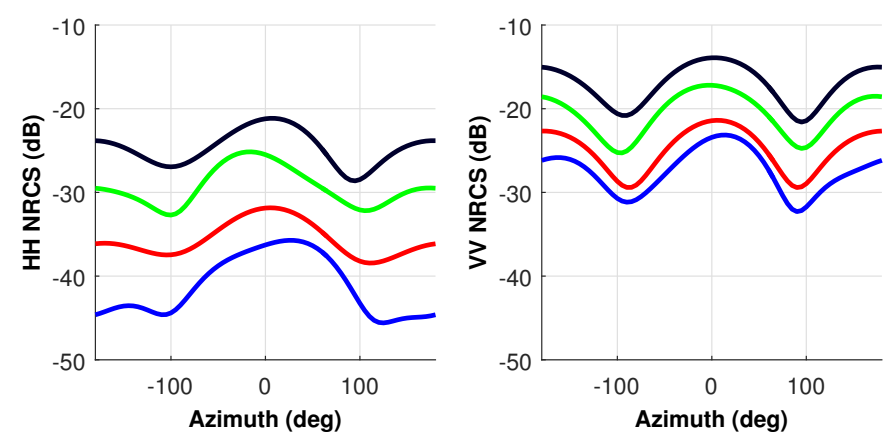

Fig. 6: Azimuthal variation in $\mathrm{HH}$ (left) and VV (right) polarizations for run day 9 with nominal grazing angles of $16^{\circ}$ (blue), $25^{\circ}$ (red), $35^{\circ}$ (green) and $45^{\circ}$ (black). Note that the azimuth axis has been rotated so that $0^{\circ}$ is upwind.

clear trend is observed. The maximum UDA in $\mathrm{HH}$ is about 2 $\mathrm{dB}$ higher than the VV counterpart, while the maximum UCA is rather insensitive to polarization and wind speed $(\sim 7 \mathrm{~dB}$ at moderate to large winds speed between $8-14 \mathrm{~m} / \mathrm{s})$. Further conclusions are difficult to make for the UDA due to the small SNR and the strong data dispersion at lower grazing angles.

These conclusions are also confirmed in Fig. 7 by examining the different azimuthal harmonics. Since the $a_{0}$ harmonic captures the NRCS mean omnidirectional value and does not contribute to directional asymmetries, the higher order harmonic amplitudes $a_{1}, \ldots, a_{4}$ have been normalized with respect to $a_{0}$. The first two harmonic components, $a_{1}$ and $a_{2}$, incorporate the most prominent contributions to the upwinddownwind and upwind-crosswind asymmetries. As clearly shown in Fig. 7, the contribution of $a_{1}$ is obviously more substantial in $\mathrm{HH}$, which supports the previously stated observation of the UDA. This trend is reversed for the $a_{2}$ harmonic, whose contribution is greater for $\mathrm{VV}$ than $\mathrm{HH}$, suggesting that the UCA asymmetry is more pronounced for VV-polarized data. The $a_{3}$ and $a_{4}$ amplitudes are small compared to $a_{1}$ and $a_{2}$, but still contribute to refining the azimuthal adjustment of the data and improving the goodness of fit.
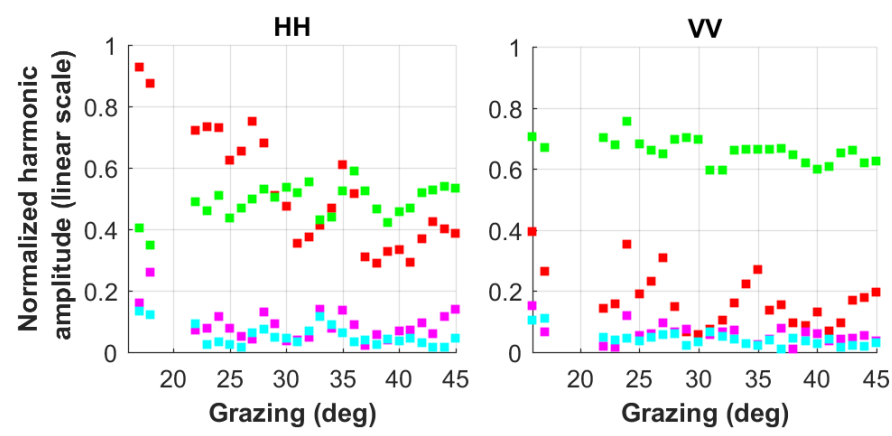

Fig. 7: Normalized harmonic amplitudes for $\mathrm{HH}$ (left) and VV (right) polarizations for run day 9: $\frac{a_{1}}{a_{0}}(\square), \frac{a_{2}}{a_{0}}(\square), \frac{a_{3}}{a_{0}}(\square)$, and $\frac{a_{4}}{a_{0}}(\square)$.

\section{RELATIONS BETWEEN THE DIFFERENT POLARIZATIONS}

To better understand the relationship between the different polarization channels, we now study the polarization ratio and difference as well as the azimuth variation of the cross polarized data.

\section{A. Polarization ratio}

The most commonly used quantity to relate the different polarization channels is the polarization ratio (PR),

$$
P R=\left(\sigma_{\mathrm{VV}}^{0}\right)_{\mathrm{dB}}-\left(\sigma_{\mathrm{HH}}^{0}\right)_{\mathrm{dB}}
$$

where the $\mathrm{dB}$ subscript indicates the result measured in decibels. An illustration of the variation with grazing angle is given in Fig. 8 for run days 9 (left) and 12 (right), where the PR is modelled using a third-order polynomial. Superimposed on this figure are the PR predicted by classical Bragg theory [23, eqns. 4.5-4.7], which is believed to be the dominant mechanism at medium grazing angles and low wind speed, as well as the PR inferred from the GOSSA model which offers a closer match to the data.

These results show the $\mathrm{PR}$ is a decreasing function with grazing and the Ingara MGA results are typically much lower than the (roughness independent) polarization ratio predicted by Bragg theory. The GO-SSA model is a closer match to the data and offers significant improvement to the PR prediction. However, it remains deficient in recovering the PR azimuthal variation (namely the upwind/downwind PR contrast and the maximum downwind PR). This discrepancy between data and models is especially true at $15^{\circ}$ grazing, where a difference of up to $10 \mathrm{~dB}$ is observed. A strong sensitivity to the azimuthal direction is also observed, with a similar PR level observed in the up and crosswind directions, while the downwind PR is systematically higher. These trends are confirmed in Fig. 9 which shows the azimuthal variation of the PR. The results show a sharp maximum around the downwind direction and a secondary maximum around the upwind direction, which can allow removing the ambiguity usually encountered between the upwind and downwind directions.
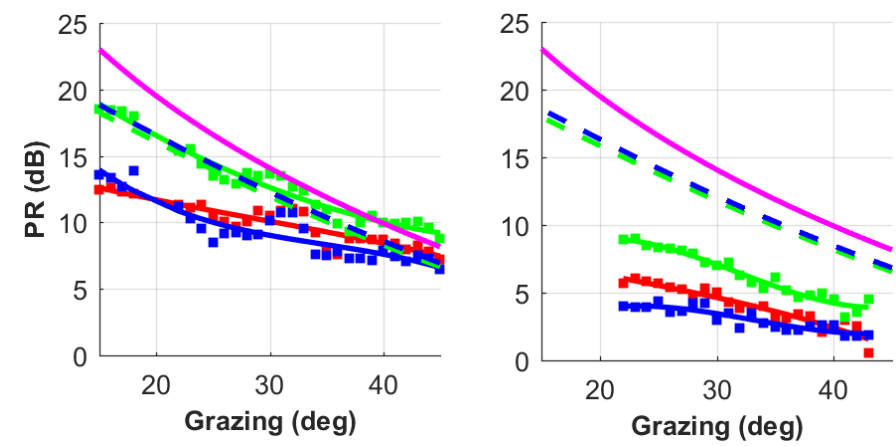

Fig. 8: Polarization ratio versus grazing angle for run days 9 (left) and 12 (right). Azimuth direction: upwind (red), crosswind (blue) and downwind (green). The magenda solid line (-) corresponds to Bragg PR and the dashed lines (- -) and (- -) to GO-SSA upwind and crosswind $\mathrm{PR}$ respectively.

\section{B. Polarization difference}

Another useful parameter is the polarization difference (PD) of the two co-polarizations using a linear scale( [24]),

$$
P D=\left(\sigma_{\mathrm{VV}}^{0}-\sigma_{\mathrm{HH}}^{0}\right)_{\mathrm{dB}}
$$

This parameter is also twice the polarized part, $\sigma_{\text {pol }}^{0}$, of the NRCS in its classical decomposition into a polarized and 

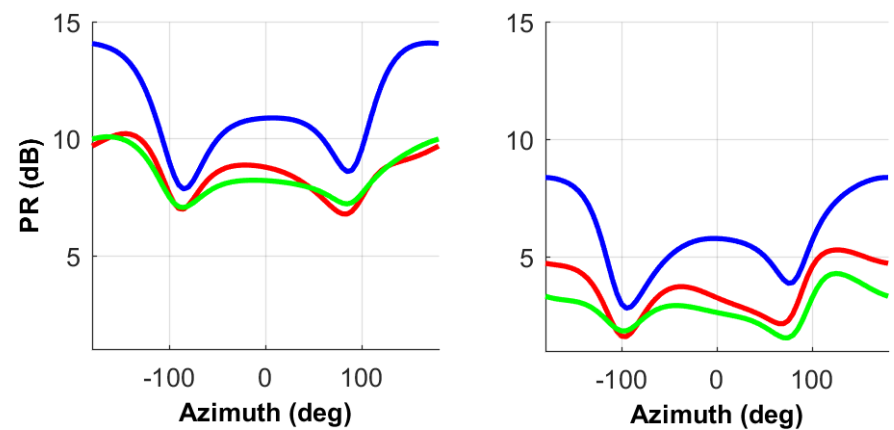

Fig. 9: Polarization ratio versus azimuth angle for run days 9 (left) and 12 (right) at nominal grazing angles of $25^{\circ}$ (blue), $37^{\circ}$ (red) and $42^{\circ}$ (green). Note that the azimuth axis has been rotated so that $0^{\circ}$ is upwind.

unpolarized part,

$$
\begin{aligned}
& \sigma_{\mathrm{VV}}^{0}=\frac{1}{2}\left(\sigma_{\mathrm{VV}}^{0}+\sigma_{\mathrm{HH}}^{0}\right)+\frac{1}{2}\left(\sigma_{\mathrm{VV}}^{0}-\sigma_{\mathrm{HH}}^{0}\right)=\sigma_{\mathrm{unpol}}^{0}+\sigma_{\mathrm{pol}}^{0}, \\
& \sigma_{\mathrm{HH}}^{0}=\frac{1}{2}\left(\sigma_{\mathrm{VV}}^{0}+\sigma_{\mathrm{HH}}^{0}\right)-\frac{1}{2}\left(\sigma_{\mathrm{VV}}^{0}-\sigma_{\mathrm{HH}}^{0}\right)=\sigma_{\mathrm{unpol}}^{0}-\sigma_{\mathrm{pol}}^{0} .
\end{aligned}
$$

It is well-known (see for example the Two-scale model or as a direct consequence of the universal weighted curvature approximation (WCA) [25, eq. 4.32]), that if one ignores the effect of the modulation by long waves, then the PD is proportional to the wave number spectrum taken at the Bragg frequency. It is therefore more sensitive to the small scale features of the sea surface, rather than the larger scales which are responsible for the unpolarized portion of the NRCS.

Figure 10 shows the azimuthal variations of the $\mathrm{PD}$ at various grazing angles for run days 9 and 12 . Note that the results are shown in $\mathrm{dB}$ scale to highlight the azimuthal PD dynamics and variations, even though the PD is calculated in a linear scale. At lower grazing angles, the PD is typically dominated by the VV component which is much stronger than $\mathrm{HH}$. However, at higher grazing angles where the VV and HH NRCS are of the same order of magnitude, the PD does not exhibit the UDA asymmetry seen with $\mathrm{HH}$ and VV polarized data. This seems to indicate that the UDA asymmetry is likely to be contained in the non-polarized part and presumably linked to the large rather than small scales of roughness. Another possibility is that breaking events, whose contribution is thought to be non-polarized, play a major role in the production of this asymmetry. This would explain why the UDA is stronger in $\mathrm{HH}$ than VV (the relative contribution of breaking being stronger in the former case).

\section{Cross-polarized data}

Similarly to the co-polarized data, we now study the azimuthal variation of the cross-polarized data. These data present an azimuthal modulation whose shape is akin to the VV-polarized data but which is remarkably less pronounced when compared to the co-polarized data in terms of asymmetry magnitudes. Figure 11 illustrates an example for the azimuthal variations of the cross-polarized for run day 9 with various grazing angles. Note that since cross-polarized measurements are usually very close to the noise floor, these conclusions on azimuthal variations have been confirmed on the higher
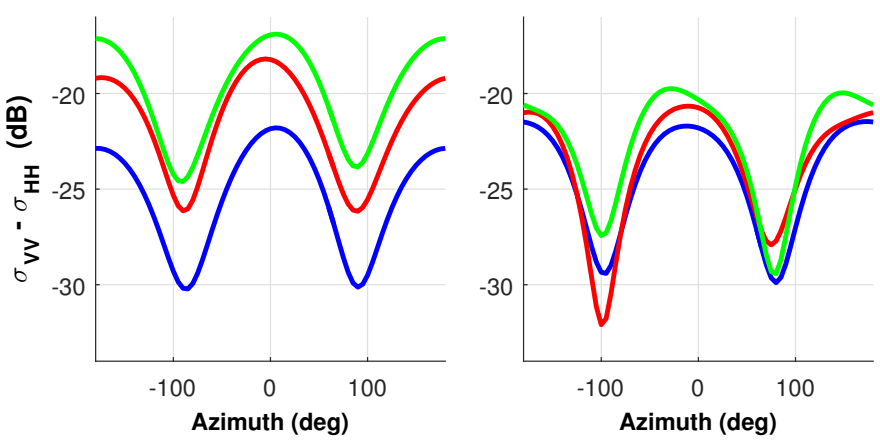

Fig. 10: Azimuthal variation of the polarization difference VV-HH for run days 9 (left) and 12 (right) at nominal grazing angles of $25^{\circ}$ (blue), $37^{\circ}$ (red) and $42^{\circ}$ (green). Note that the azimuth axis has been rotated so that $0^{\circ}$ is upwind.

grazing angle HV data where the SNR is good enough to assign confidence to the results.

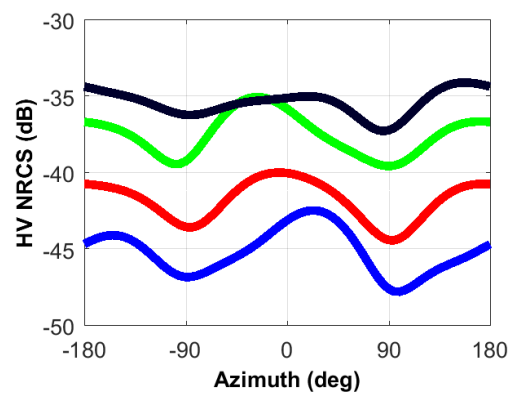

Fig. 11: Azimuthal variation in HV polarization for run day 9 with nominal grazing angles of $16^{\circ}$ (blue), $25^{\circ}$ (red), $35^{\circ}$ (green) and $45^{\circ}$ (black). Note that the azimuth axis has been rotated so that $0^{\circ}$ is upwind.

We now investigate the azimuthal dependence of the crosspolarized NRCS (VH or HV) in the light of a recent simplified formulation [26] of the second-order small-slope approximation (SSA2), [27]. In this work, a numerically efficient and almost equivalent version of SSA2 was developed for the cross-polarized backscatter. It was shown that, starting about $30^{\circ}$ away from nadir, the cross-polarized component, $\sigma_{H V}^{0}$, is well approximated by a quantity proportional to the wave number spectrum taken at the Bragg frequency, $\Gamma_{B}$ times the radar-filtered directional mean square slope, $\mathrm{mss}_{y}$ crosswise to the radar line-of-sight.

In addition, since the PD is also proportional to $\Gamma_{B}$, apart from a geometric coefficient, the following ratio is essentially proportional to the aforementioned mean square slope:

$$
R=\frac{\sigma_{H V}^{0}}{\sigma_{\mathrm{VV}}^{0}-\sigma_{\mathrm{HH}}^{0}} \propto \operatorname{mss}_{y} .
$$

Hence the above ratio should reflect the azimuthal variations of the $\operatorname{mss}_{y}$ parameter, which is maximum whenever the radar is perpendicular to the wind direction (in which case $\operatorname{mss}_{y}$ is the upwind mean square slope).

To verify this qualitative prediction, the ratio $R$ has been systematically computed from the Ingara MGA data set for different grazing angles and sea states. The azimuthal variations of $R$ are shown in Fig. 12 at $40^{\circ}$ grazing angle for run days 3 and 9 , and are in qualitative agreement with 
the model prediction showing a pronounced maximum in the crosswind direction. For further verification, a quantitative comparison has been performed with two different spectral models combined with the SSA2 scattering model. The first is the classical directional Elfouhaily spectral model, while the second is a more recent directional spectral model combining the omnidirectional spectrum proposed in [28] based on remote sensing data (Bringer model) and the angular spreading function proposed in [29] based on stereographic measurements (Yuroskaya model). The combined use of the Bringer omnidirectional spectrum and the Yuroskaya angular spreading function will be referred to as the Bringer-Yuroskaya (BY) model. As shown in Fig. 12, the azimuthal variations of the data are retrieved but are stronger than those predicted by the model. This means that the heuristic arguments which led to the formula V.9 are not sufficiently accurate to evaluate $R$ even though they give the correct trend.
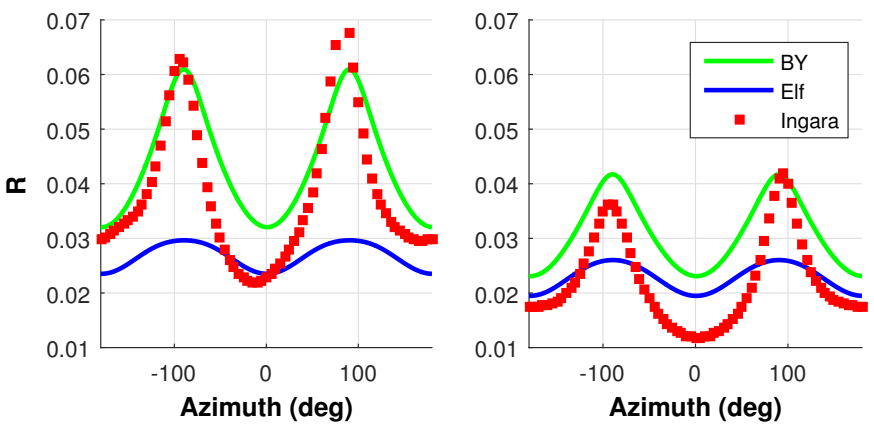

Fig. 12: Azimuthal variation of the $R$ ratio in (V.9) at $40^{\circ}$ grazing angle for run days 3 (left) and 9 (right). Note that the azimuth axis has been rotated so that $0^{\circ}$ is upwind.

\section{MODEL COMPARISON}

In this last section, we evaluate the performance of some currently available sea surface scattering models with the Ingara MGA sea clutter data set.

\section{A. Choice of the spectral model}

The co-polarized channels of the NRCS have been estimated with the improved Two-Scale model [22] referred to as 'GOSSA' as it is based on a combination of the Geometrical Optics and the Small-Slope Approximation. In this calculation, both Elfouhaily and Bringer-Yuroskaya spectral models have been used for the different wind speeds reported in Table I. These spectral models have also been used to estimate the crosspolarized channels with the help of the previously introduced numerical version of the SSA2 model. The results shown in Figs. 13-15 demonstrate that the recent BY spectral model brings significant improvement to the simulation of the NRCS. For the co-polarized channels, it is in close agreement with data for both upwind and crosswind directions. However, due to the elevated noise floor in the cross-polarized channel, a significant comparison can only be drawn in the upwind direction, where the agreement with the BY model is satisfactory.

The same conclusions are drawn from plots of azimuthal variation of Ingara data and associated model comparison. Although upwind/downwind as well as crosswind/crosswind asymmetries are not captured by centro-symmetric models (which results in azimuthal discrepancy between data and models especially when data exhibit strong asymmetries), the improvement brought by BY model is clearly shown in Fig. 14.
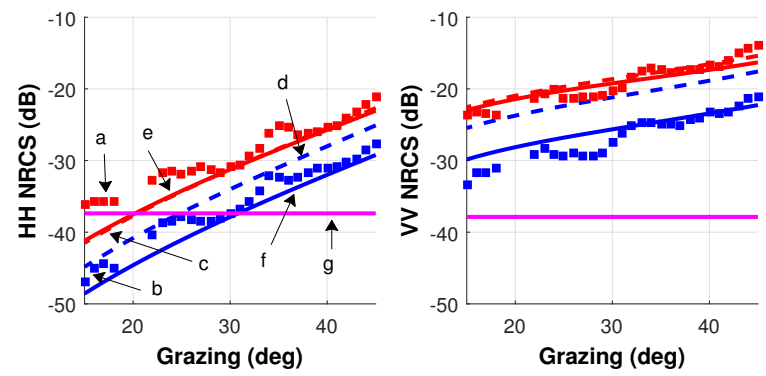

Fig. 13: HH (left) and VV (right) NRCS from the Ingara MGA data for

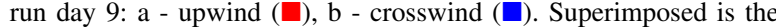
simulated NRCS according to the GO-SSA model with Elfouhaily directional spectrum: $\mathrm{c}$ - upwind (- -), d - crosswind (- -) and BringerYurovskaya model: e - upwind (-), f - crosswind (-). Mean noise estimate is shown in $\mathrm{g}$ - magenta.
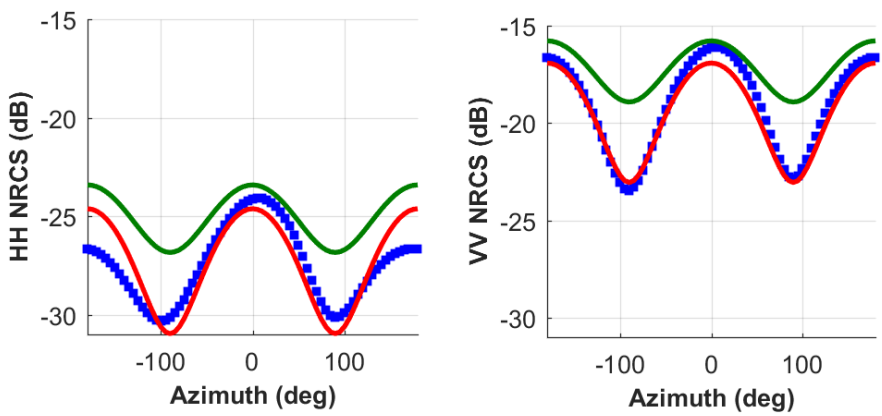

Fig. 14: $H H$ (left) and VV (right) azimuthal NRCS from the Ingara MGA data (ם) for run day 9 at nominal grazing angle of $42^{\circ}$. Superimposed is the simulated NRCS according to the GO-SSA model with Elfouhaily directional spectrum (-) and Bringer-Yurovskaya model $(-)$.

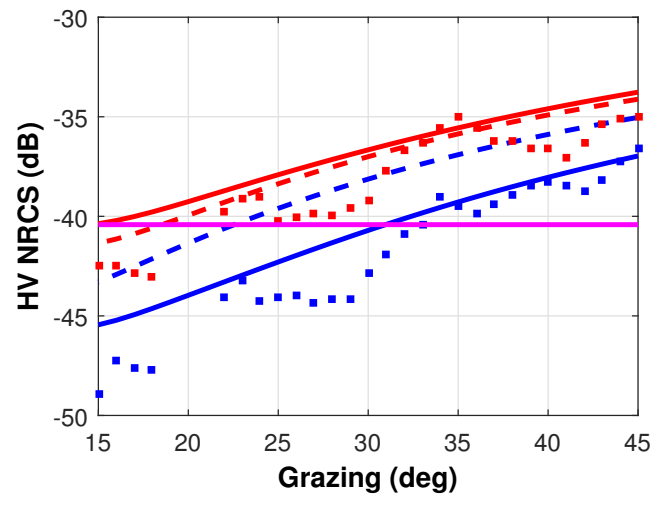

Fig. 15: Same as Fig. 13 for the HV NRCS. 


\section{B. Effect of the swell}

In the previous model comparison, the contribution of swell has been ignored. However, the sometimes important values of wave height reported in Table I show that a strong swell is present for most run days of the Ingara MGA data set. To quantify the contribution of swell we relied on the measurements reported in [30] where the sea spectrum has been evaluated for different wind speeds and classified according to four swell indices. The GO-SSA sea surface scattering model has been combined with these different spectra to study the sensitivity of the NRCS to the swell component. The NRCS calculated at X-band with the different swell indices is found to differ by at most $2 \mathrm{~dB}$ for $\mathrm{HH}$ and $1.5 \mathrm{~dB}$ for $\mathrm{VV}$ at the smallest wind speed and by $1.5 \mathrm{~dB}$ for $\mathrm{HH}$ and $1 \mathrm{~dB}$ for $\mathrm{VV}$ at the largest wind speed. As expected, the effect of swell is slightly more pronounced in the $\mathrm{HH}$ polarization (which is more sensitive to the long wave tilting effect) and at smaller wind speeds (where the relative contribution of the swell to the mean square slope is larger). These results can also be confirmed by performing a simple numerical simulation of a monochromatic swell with a wave height and period matching the Ingara data. Assuming the GO-SSA model, the swell solely affects the low frequency slope modulation and the swell mean square slope slightly modifies the total mean square slope. As a result, the difference between $m s s_{x}$ and $m s s_{y}$ yields a change in the azimuthal NRCS and since the swell slopes are relatively small, its impact on the azimuthal variation is shown to be very small and slightly more pronounced in $\mathrm{HH}$ polarization. Therefore, given the dispersion of Ingara MGA data, the 1-2 $\mathrm{dB}$ possible contribution of swell discussed above can be ignored when assessing model comparisons at X-band.

\section{CONCLUSION}

In this work, we have undertaken a thorough analysis of the geometric and polarimetric properties of the Ingara MGA sea clutter data set and introduced a novel probabilistic denoising procedure introduced for the low SNR regions. The data analysis has been complemented with a comparison of modern sea surface scattering models to evaluate their suitability for modelling real MGA sea clutter. Our main findings can be summarized as follows:

- The azimuthal behavior of the HH NRCS was confirmed at both medium and low grazing angles, with the latter demonstrating the vanishing of the secondary downwind maximum.

- The directional asymmetries were strongly polarization dependent and exhibited non-monotonic variations with respect to the grazing angle. The maximum UDA and UCA in the two co-polarizations lie at moderate grazing angles between about $35^{\circ}$ and $45^{\circ}$ with little sensitivity to wind speed. The HH NRCS favors the upwind/downwind asymmetry with a maximum UDA approximately $2 \mathrm{~dB}$ higher than the VV NRCS, while the maximum UCA did not exhibit clear variations with the polarization and wind speed, remaining around $7 \mathrm{~dB}$ at moderate to large wind speeds between $8-14 \mathrm{~m} / \mathrm{s}$.
- The polarization ratio was strongly dependent on the radar collection geometry and showed very different results at lower grazing angles than the value predicted from Bragg theory. The PR variation with respect to azimuth angle presented a sharp maximum in the downwind direction. Furthermore, we showed that the polarization difference between the two co-polarized NRCS channels has a much lower UDA than the HH and VV polarized NRCS, suggesting that the non-polarized NRCS (and therefore large scale wave structures or possibly breaking events) play a major role in the production of this asymmetry.

- For model assessment purposes, the GO-SSA model and a simplified version of the SSA2 model have been used to estimate the NRCS of the co- and cross-polarizations. A strong discrepancy between the model predictions and observations have been found in the case of the classical Elfouhaily directional spectrum. More consistent results were obtained by combining the recent spectral models of Bringer and Yurovskaya.

- In order to evaluate the impact of swell on the NRCS at X-band, we have combined the GO-SSA model with experimental spectra reported in [30], where different swell magnitudes were available. The variability induced by the presence of a swell is estimated to be of the order of 1 to $2 \mathrm{~dB}$. It was more pronounced for low wind speeds and for the $\mathrm{HH}$ polarization, but remains negligible regarding the margin of fluctuations in the Ingara MGA data set.

\section{ACKNOWLEDGMENTS}

The Région Provence-Alpes-Côte d'Azur (PACA) and the ONERA are acknowledged for funding the $\mathrm{PhD}$ of $\mathrm{Z}$. Guerraou. Special thanks go to the Defence Science and Technology Group for providing the Ingara MGA data set and to Paul Hwang for providing us with sea spectra in the presence of swell.

\section{REFERENCES}

[1] T. Elfouhaily and C. A. Guérin. A critical survey of approximate scattering wave theories from random rough surfaces. Waves in Random and Complex Media, 14(4):1-40, 2004.

[2] G. J. Komen, L. Cavaleri, M. Donelan, K. Hasselmann, S. Hasselmann, and P. A. E. M. Janssen. Dynamics and modelling of ocean waves. Cambridge university press, 1996.

[3] T. Elfouhaily, B. Chapron, K. Katsaros, and D. Vandemark. A unified directional spectrum for long and short wind-driven waves. J. Geophys. Res., 102(C7):15781-15796, July 1997.

[4] P. Janssen. The interaction of ocean waves and wind. Cambridge University Press, 2004.

[5] P.-Y. Le Traon, D. Antoine, A. Bentamy, H. Bonekamp, L. A. Breivik, B. Chapron, G. Corlett, G. Dibarboure, P. DiGiacomo, and C. Donlon et. al. Use of satellite observations for operational oceanography: recent achievements and future prospects. Journal of Operational Oceanography, 8(sup1):s12-s27, 2015.

[6] S. Tanelli, S. L. Durden, and E. Im. Simultaneous measurements of $\mathrm{Ku}$-and Ka-band sea surface cross sections by an airborne radar. IEEE Geoscience and Remote Sensing Letters, 3(3):359-363, 2006.

[7] A. A. Mouche, B. Chapron, N. Reul, D. Hauser, and Y. Quilfen. Importance of the sea surface curvature to interpret the normalized radar cross section. J. Geophys. Res., 112(C10):10002, 2007. 
[8] N. Majurec, J. T. Johnson, S. Tanelli, and S. L. Durden. Comparison of model predictions with measurements of $\mathrm{Ku}$ - and $\mathrm{Ka}$-band near-nadir normalized radar cross sections of the sea surface from the genesis and rapid intensification processes experiment. IEEE Transactions on Geoscience and Remote Sensing, 52(9):5320-5332, Sept 2014.

[9] G. Caudal, D. Hauser, R. Valentin, and C. Le Gac. KuROS: A new airborne Ku-band Doppler radar for observation of surfaces. Journal of Atmospheric and Oceanic Technology, 31(10):2223-2245, 2014.

[10] S. Angelliaume, V. Fabbro, G. Soriano, and C. A. Guerin. The gossa extended model for all-incidence sea clutter modeling. Geoscience and Remote Sensing Symposium (IGARSS), 2014 IEEE International, Quebec City, QC, pp. 5017-5020, 2014.

[11] A. G. Voronovich and V. U. Zavorotny. Theoretical model for scattering of radar signals in $\mathrm{Ku}$-and $\mathrm{C}$-bands from a rough sea surface with breaking waves. Waves in Random Media, 11(3):247-269, 2001.

[12] H. Johnsen, G. Engen, and G. Guitton. Sea-surface polarization ratio from Envisat ASAR AP data. IEEE Transactions on Geoscience and Remote Sensing, 46(11):3637-3646, 2008.

[13] A. Bringer, C.-A. Guérin, B. Chapron, and A. Mouche. Peakedness effects in near-nadir radar observations of the sea surface. IEEE Transactions on Geoscience and Remote Sensing, 50(9):3293-3301, 2012.

[14] L. Rosenberg and S. Watts. High grazing angle sea-clutter literature review. General Document DSTO-GD-0736, DSTO, 2013.

[15] F. E. Nathanson, J. P. Reilly, and M. N. Cohen. Radar design principlessignal processing and the environment. 91, 1991.

[16] F. T. Ulaby, R. K. Moore, and A. K. Fung. Microwave remote sensing: Active and passive. Volume II: Radar Remote Sensing and Surface Scattering and Emission Theory, 1982.

[17] D. J. Crisp, R. Kyprianou, L. Rosenberg, and N. J. Stacy. Modelling Xband sea clutter at moderate grazing angles. IEEE International Radar Conference, pages 596-601, 2008.

[18] M.M. Horst, F.B. Dyer, and M.T. Tuley. Radar sea clutter model. Int. Conf. on Antennas and propagation, IEE Conf: Pub.169, 1978.

[19] N. J. S. Stacy, D. Crisp, A. Goh, D. Badger, and M. Preiss. Polarimetric analysis of fine resolution X-band SAR sea clutter data. In IEEE Geoscience and Remote Sensing Symposium, volume 4, pages 27872790, 2005.

[20] L. Rosenberg, D. J. Crisp, and N. J. Stacy. Analysis of high grazing angle sea-cluter with the KK-distribution. Technical Report DSTO-TR2915, DSTO, November 2013.

[21] C. Bourlier. Azimuthal harmonic coefficients of the microwave backscattering from a non-gaussian ocean surface with the first-order ssa model. Geoscience and Remote Sensing, IEEE Transactions on , vol.42, no.11, pp.2600,2611, Nov. 2004.

[22] G. Soriano and C. A. Guérin. A cutoff invariant two-scale model in electromagnetic scattering from sea surfaces. IEEE Geoscience and Remote Sensing Letters, 5(2):199-203, 2008.

[23] G. R. Valenzuela. Theories for the interaction of electromagnetic and oceanic waves- a review. Boundary-Layer Meteorology, 13(1):61-85, 1978.

[24] V.N. Kudryavtsev, B. Chapron, A.G. Myasoedov, F. Collard, and J.A Johannessen. On dual co-polarized sar measurements of the ocean surface. Geoscience and Remote Sensing Letters, IEEE , vol.10, no.4, pp.761,765, July 2013.

[25] C.-A. Guérin, G. Soriano, and B. Chapron. The weighted curvature approximation in scattering from sea surfaces. Waves in Random and Complex Media, 20(3):364-384, 2010.

[26] C.-A. Guérin and J.-T. Johnson. A simplified formulation for the crosspolarized backscattering coefficient under the second-order small slope approximation. IEEE Trans. Geosci. and Remote Sens., 2015. submitted.

[27] A. Voronovich. Small-slope approximation for electromagnetic wave scattering at a rough interface of two dielectric half-spaces. Waves in Random and Complex Media, 4(3):337-367, 1994.

[28] A. Bringer, B. Chapron, A. Mouche, and C.-A. Guérin. Revisiting the short-wave spectrum of the sea surface in the light of the weighted curvature approximation. IEEE Trans. Geosci. and Remote Sens., 52(1):679-689, 2014.

[29] M. V. Yurovskaya, V. A. Dulov, B. Chapron, and V. N. Kudryavtsev. Directional short wind wave spectra derived from the sea surface photography. Journal of Geophysical Research: Oceans, 118(9):4380 4394, 2013.

[30] P. A. Hwang. Observations of swell influence on ocean surface roughness. Journal of Geophysical Research: Oceans (1978-2012), 113(C12), 2008. 